\title{
On uniformly univalent functions with respect to symmetrical points
}

\section{Khalida Inayat Noor}

\section{"Correspondence:}

khalidan@gmail.com

Department of Mathematics, COMSATS Institute of Information Technology, Park Road, Islamabad, Pakistan

\begin{abstract}
In this paper, we define and study some new subclasses of starlike and close-to-convex functions with respect to symmetrical points. These functions map the open unit disc onto certain conic regions in the right half plane. Some basic properties, a necessary condition, and coefficient and arc length problems are investigated. The mapping properties of the functions in these classes are studied under a certain linear operator.
\end{abstract}

MSC: $30 C 45 ; 30 C 50$

Keywords: conic domains; starlike; close-to-convex; univalent; uniformly convex; coefficient; integral operator

\section{Introduction}

Let $A$ be the class of functions of the form

$$
f(z)=z+\sum_{n=2}^{\infty} a_{n} z^{n},
$$

which are analytic in the open unit disc $E=\{z:|z|<1\}$. Let $S, K, S^{*}$, and $C$ be the subclasses of $A$ which consist of univalent, close-to-convex, starlike (with respect to origin), and convex functions, respectively. For recent developments, extensions, and applications, see [1-25] and the references therein.

A function $f$ in $A$ is said to be uniformly convex in $E$ if $f$ is a univalent convex function along with the property that, for every circular $\operatorname{arc} \gamma$ contained in $E$, with center $\xi$ also in $E$, the image curve $f(\gamma)$ is a convex arc. The class of uniformly convex functions is denoted by $U C V$. The corresponding class $U S T$ is defined by the relation that $f \in U C V$ if, and only if, $z f^{\prime} \in U S T$. It is well known [13] that $f \in U C V$ if, and only if

$$
\left|\frac{z f^{\prime \prime}(z)}{f^{\prime}(z)}\right|<\Re\left\{1+\frac{z f^{\prime \prime}(z)}{f^{\prime}(z)}\right\} \quad(z \in E) .
$$

Uniformly starlike and convex functions were first introduced by Goodman [3] and then studied by various other authors. If $f, g \in A$, we say $f$ is subordinate to $g$ in $E$, written as $f \prec g$ or $f(z) \prec g(z)$, if there exists a Schwarz function $w(z)$ such that $f(z)=g(w(z))$ for $z \in E$.

@2014 Noor; licensee Springer. This is an Open Access article distributed under the terms of the Creative Commons Attribution License (http://creativecommons.org/licenses/by/2.0), which permits unrestricted use, distribution, and reproduction in any medium, provided the original work is properly cited. 
For $0 \leq \beta<1$, the class $P(\beta)$ consists of functions $p(z)$ analytic in $E$ with $p(0)=1$ such that $\Re p(z)>\beta$ for $z \in E$, and, with $\beta=0$, we obtain the well-known class $P$ of Carathéodory functions with positive real part.

For $k \in[0, \infty)$, the conic regions $\Omega_{k}$ are defined as follows, see [5]:

$$
\Omega_{k}=\left\{u+i v: u>k \sqrt{(u-1)^{2}+v^{2}}\right\} .
$$

For fixed $k, \Omega_{k}$ represents the conic regions bounded, successively, by the imaginary axis $(k=0)$, the right branch of a hyperbolic $(0<k<1)$ and a parabola $v^{2}=2 u-1(k=1)$. When $k>1$, the domain becomes a bounded domain being the interior of the ellipse.

We shall consider the case when $k \in[0,1]$. Related to the domain $\Omega_{k}$, the following functions $p_{k}(z), k \in[0,1]$, play the role of extremal functions mapping in $E$ onto $\Omega_{k}$ :

$$
p_{k}(z)= \begin{cases}\frac{1+z}{1-z} & (k=0), \\ 1+\frac{2}{\pi^{2}}\left(\log \frac{1+\sqrt{z}}{1-\sqrt{z}}\right)^{2} & (k=1), \\ 1+\frac{2}{1-k^{2}} \sinh ^{2}\left[\left(\frac{2}{\pi} \arccos k\right) \operatorname{arctanh} \sqrt{z}\right] & (0<k<1) .\end{cases}
$$

These functions are univalent in $E$ and belong to the class $P$. Using the subordination concept, we define the class $P\left(p_{k}\right)$ as follows.

Let $p(z)$ be analytic in $E$ with $p(0)=1$. Then $p \in P\left(p_{k}\right)$ if, and only if, $p \prec p_{k}$ in $E$ and $p_{k}(z)$ are given by (1.2).

The conic domains $\Omega_{k}$ can be generalized as given by

$$
\Omega_{k, \beta}=(1-\beta) \Omega_{k}+\beta,
$$

with the corresponding extremal function

$$
p_{k, \beta}(z)=(1-\beta) p_{k}+\beta \quad(0 \leq \beta<1, k \in[0,1]) .
$$

It can easily be seen that the analytic function $p(z)$, with $p(0)=1$, belongs to the class $P\left(p_{k, \beta}\right)$ if $p(z) \prec p_{k, \beta}(z)$ in $E$.

It is easy to verify that $P\left(p_{k, \beta}\right)$ is a convex set. It is known [6] that

$$
P\left(p_{k}\right) \subset P\left(\frac{k}{k+1}\right) \subset P
$$

and, for $p \in P\left(p_{k}\right)$, we have

$$
|\arg p(z)| \leq \sigma \frac{\pi}{2}
$$

where

$$
\sigma=\frac{2}{\pi} \arctan \frac{1}{k} .
$$

So we can write $p(z)=h^{\sigma}(z), h \in P$. 
Also

$$
P\left(p_{k, \beta}\right) \subset P\left(\frac{k+\beta}{k+1}\right) \subset P .
$$

Sakaguchi [24] introduced and studied the class $S_{s}^{*}$ of starlike functions with respect to symmetrical points. The class $S_{s}^{*}$ includes the classes of convex and odd starlike functions with respect to the origin. It was shown [24] that a necessary and sufficient condition for $f \in S_{s}^{*}$ to be univalent and starlike with respect to symmetrical points in $E$ is that

$$
\left(\frac{2 z f^{\prime}(z)}{f(z)-f(-z)}\right) \in P, \quad z \in E
$$

Das and Singh [2] defined the classes $C_{s}$ of convex functions with respect to symmetrical points and showed that a necessary and sufficient condition for $f \in C_{s}$ is that

$$
\frac{2\left(z f^{\prime}(z)\right)^{\prime}}{(f(z)-f(-z))^{\prime}} \in P, \quad z \in E
$$

It is also well known [2] that $f \in C_{s}$ if, and only if, $z f^{\prime} \in S_{s}^{*}$.

We now define the following.

Definition 1.1 Let $f \in A$. The $f$ is said to be in the class $k-S T_{s}(\beta)$ if, and only if,

$$
\frac{2 z f^{\prime}(z)}{(f(z)-f(-z))} \in P\left(p_{k, \beta}\right), \quad z \in E
$$

It can easily be seen that

$$
k-S T_{s}(\beta) \subset S_{s}^{*} \subset S_{s}^{*}, \quad \beta_{1}=\frac{k+\beta}{k+1} .
$$

Also, for $\beta=0=k$, the class $k-S T_{s}(\beta)$ reduces to $S_{s}^{*}$.

The class $k-U C V_{s}(\beta)$ is defined as follows.

Definition 1.2 Let $f \in A$. Then $f \in k-U C V_{s}(\beta)$ if, and only if $z f^{\prime} \in k-S T_{s}(\beta)$ for $z \in E$.

We note that

$$
k-U C V_{s}(\beta) \subset C_{s}\left(\beta_{1}\right) \subset C_{s}, \quad \beta_{1}=\frac{k+\beta}{k+1} .
$$

Definition 1.3 Let $f \in A$. Then $f \in k-U K_{s}(\beta)$ if, and only if, there exists $g \in k-S T_{s}(\beta)$ such that

$$
\left(\frac{2 z f^{\prime}(z)}{g(z)-g(-z)}\right) \in P\left(p_{k, \beta}\right), \quad z \in E
$$

Since $P\left(p_{k, \beta}\right) \subset P\left(\beta_{1}\right) \subset P, \beta_{1}=\frac{k+\beta}{k+1}$, and $k-S T_{s}(\beta) \subset S_{s}^{*}$, we note that

$$
k-U K_{s}(\beta) \subset K_{s} \subset K,
$$


where $k_{S}$ consists of close-to-convex functions with respect to symmetrical starlike functions.

From the definition, it is clear that $k-U K_{s}(\beta)$ consists of univalent functions.

For $k=0, \beta=0$ and $f(z)=g(z), k-U K_{s}(\beta)$ reduces to the class $S_{s}^{*}$.

\section{Preliminary results}

We shall need the following lemmas to prove our main results.

Lemma 2.1 [15] Let $q(z)$ be a convex function in $E$ with $q(0)=1$ and let another function $h: E \rightarrow \mathbb{C}$ be with $\Re h(z)>0$. Let $p(z)$ be analytic in $E$ with $p(0)=1$ such that

$$
\left(p(z)+h(z) z p^{\prime}(z)\right) \prec q(z), \quad z \in E .
$$

Then $p(z) \prec q(z), z \in E$.

Lemma 2.2 Let $N(z), D(z)$ be analytic in $E$ with

$$
N(0)=0=D(z)
$$

and let $D \in S^{*}$ for $z \in E$. Then $\frac{N^{\prime}(z)}{D^{\prime}(z)} \in P\left(p_{k, \beta}\right)$ implies that $\frac{N(z)}{D(z)} \in P\left(p_{k, \beta}\right)$ for $z \in E$.

Proof Let

$$
\frac{N(z)}{D(z)}=p(z)
$$

Then

$$
\frac{N^{\prime}(z)}{D^{\prime}(z)}=p(z)+h(z)\left(z p^{\prime}(z)\right), \quad h(z)=\frac{1}{h_{0}(z)},
$$

where

$$
h_{0}(z)=\frac{z D^{\prime}(z)}{D(z)} \in P \text {. }
$$

Since $\frac{N^{\prime}(z)}{D^{\prime}(z)} \in P\left(p_{k, \beta}\right)$, we have

$$
\frac{N^{\prime}(z)}{D^{\prime}(z)}=\left(p(z)+h(z)\left(z p^{\prime}(z)\right)\right) \prec p_{k, \beta}(z), \quad z \in E .
$$

We now use Lemma 2.1 and this implies that

$$
\frac{N(z)}{D(z)}=p(z) \prec p_{k, \beta}(z) \quad \text { in } E .
$$

This proves that $\frac{N(z)}{D(z)} \in P\left(p_{k, \beta}\right)$ for $z \in E$.

The following lemma is an easy extension of a result proved in [5]. 
Lemma 2.3 Let $k \in[0, \infty)$ and $\gamma_{1}, \delta_{1}$ be any complex numbers with $\gamma_{1} \neq 0$ and let $\Re\left\{\frac{\gamma_{1} k}{k+1}+\right.$ $\left.\delta_{1}\right\}>\beta$. If $h(z)$ is analytic in $E, h(0)=1$ and it satisfies

$$
\left(h(z)+\frac{z h^{\prime}(z)}{\gamma_{1} h(z)+\delta_{1}}\right) \prec p_{k, \beta}(z)
$$

and $q_{k, \beta}(z)$ is an analytic solution of

$$
\left(q_{k, \beta}(z)+\frac{z q_{k, \beta}^{\prime}(z)}{\gamma_{1} q_{k, \beta}(z)+\delta_{1}}\right)=p_{k, \beta}(z)
$$

then $q_{k, \beta}$ is univalent and

$$
h(z) \prec q_{k, \beta}(z) \prec p_{k, \beta}(z),
$$

and $q_{k, \beta}(z)$ is the best dominant of $(2.1)$.

\section{The class $k-S T_{s}(\beta)$}

In this section, we shall study some basic properties of the class $k-S T_{s}(\beta)$.

Theorem 3.1 Let $f \in k-S T_{s}(\beta)$. Then the odd function

$$
\Psi(z)=\frac{1}{2}[f(z)-f(-z)]
$$

belongs to $k-S T(\beta)$ in $E$.

In particular $\Psi(z)$ is an odd starlike function of order $\beta_{1}=\frac{k+\beta}{k+1}$ in $E$.

Proof Logarithmic differentiation of (3.1) and simple computation yield

$$
\begin{aligned}
\frac{z \Psi^{\prime}(z)}{\Psi(z)} & =\frac{1}{2}\left[\frac{2 z f^{\prime}(z)}{f(z)-f(-z)}+\frac{2(-z) f^{\prime}(-z)}{f(-z)-f(z)}\right] \\
& =\frac{1}{2}\left[p_{1}(z)+p_{2}(z)\right], \quad \text { for } z \in E, p_{1}, p_{2} \in P\left(p_{k, \beta}\right) .
\end{aligned}
$$

Since $P\left(p_{k, \beta}\right)$ is a convex set, it follows that $\frac{z \Psi^{\prime}(z)}{\Psi(z)} \in P\left(p_{k, \beta}\right)$ and thus $\Psi \in k-S T(\beta)$ in $E$.

As a special case, we note that, for $k=0=\beta, \frac{1}{2}[f(z)-f(-z)]=\Psi(z) \in S^{*}$ in $E$, and hence $\frac{z f^{\prime}}{\Psi} \in P$. We now discuss a geometric property for $f \in k-S T_{s}(\beta)$. Here we investigate the behavior of the inclusion of the tangent at a point $w(\theta)=f\left(r e^{i \theta}\right)$ to the image $\Gamma_{r}$ of the circle $C_{r}=\{z:|z|=r\}, 0 \leq r<1, \theta \in[0,2 \pi]$, under the mapping by means of a function $f$ from the class $f \in k-S T_{s}(\beta)$.

Let

$$
\Phi(\theta)=\frac{\pi}{2}+\theta+\arg f^{\prime}\left(r e^{i \theta}\right)=\arg \frac{\partial}{\partial \theta} f\left(r e^{i \theta}\right)
$$


and, for $\theta_{2}>\theta_{1}, \theta_{1}, \theta_{2} \in[0,2 \pi]$,

$$
\Phi\left(\theta_{2}\right)-\Phi\left(\theta_{1}\right)=\theta_{2}+\arg f^{\prime}\left(r e^{i \theta_{2}}\right)-\theta_{1}-\arg f^{\prime}\left(r e^{i \theta_{1}}\right) .
$$

Now, since

$$
\theta+\arg f^{\prime}\left(r e^{i \theta}\right)=\theta+\Re\left\{-i \ln f^{\prime}\left(r e^{i \theta}\right)\right\},
$$

then

$$
\frac{\partial}{\partial \theta}\left(\theta+\arg f^{\prime}\left(r e^{i \theta}\right)\right)=\Re\left\{1+\frac{r e^{i \theta} f^{\prime \prime}\left(r e^{i \theta}\right)}{f^{\prime}\left(r e^{i \theta}\right)}\right\} .
$$

Hence

$$
\int_{\theta_{1}}^{\theta_{2}} \frac{\partial}{\partial \theta}\left(\theta+\arg f^{\prime}\left(r e^{i \theta}\right)\right) d \theta=\int_{\theta_{1}}^{\theta_{2}} \Re\left\{1+\frac{r e^{i \theta} f^{\prime \prime}\left(r e^{i \theta}\right)}{f^{\prime}\left(r e^{i \theta}\right)}\right\} d \theta .
$$

Also, on the other hand,

$$
\begin{aligned}
\int_{\theta_{1}}^{\theta_{2}} \frac{\partial}{\partial \theta}\left(\theta+\arg f^{\prime}\left(r e^{i \theta}\right)\right) d \theta & =\theta_{2}+\arg f^{\prime}\left(r e^{i \theta_{2}}\right)-\theta_{1}-\arg f^{\prime}\left(r e^{i \theta_{1}}\right) \\
& =\Phi\left(\theta_{2}\right)-\Phi\left(\theta_{1}\right) .
\end{aligned}
$$

So, the integral on the left side of the last inequality characterizes the increment of the angle of the inclination of the tangent to the curve $\Gamma_{r}$ between the points $w\left(\theta_{2}\right)$ and $w\left(\theta_{1}\right)$ for $\theta_{2}>\theta_{1}$.

We have the following necessary condition for $f \in k-S T_{s}(\beta)$.

Theorem 3.2 Let $f \in k-S T_{s}(\beta)$. Then, with $z=r e^{i \theta}$ and $0 \leq \theta_{1}<\theta_{2} \leq 2 \pi, 0 \leq \beta<1$ and $0 \leq k \leq 1$, we have

$$
\int_{\theta_{1}}^{\theta_{2}} \Re\left\{\frac{\left(z f^{\prime}(z)\right)^{\prime}}{f^{\prime}(z)}\right\} d \theta>-\sigma \pi+2 \cos ^{-1}\left\{\frac{2(1-\beta)}{1-(1-2 \beta) r^{2}}\right\}+\beta_{1}\left(\theta_{2}-\theta_{1}\right),
$$

where $\sigma$ is given by (1.3) and $\beta_{1}=\frac{k+\beta}{k+1}$.

Proof Since $\frac{f^{\prime}(z)}{\Psi^{\prime}(z)} \in P\left(p_{k, \beta}\right), \Psi(z)=\frac{1}{2}[f(z)-f(-z)]$ and $\Psi \in k-U C V(\beta) \subset C(\beta)$.

We can write

$$
f^{\prime}(z)=\left(\Psi_{1}^{\prime}(z)\right)^{1-\beta_{1}} h^{\sigma}(z), \quad \Psi_{1} \in C, h \in P(\beta),
$$

and this gives us, with $z=r e^{i \theta}, 0 \leq r<1,0 \leq \theta_{1}<\theta_{2} \leq 2 \pi$,

$$
\begin{aligned}
\int_{\theta_{1}}^{\theta_{2}} \Re\left\{\frac{\left(z f^{\prime}(z)\right)^{\prime}}{f^{\prime}(z)}\right\} d \theta= & \left(1-\beta_{1}\right) \int_{\theta_{1}}^{\theta_{2}} \Re\left\{\frac{\left(z \Psi_{1}^{\prime}(z)\right)^{\prime}}{\Psi_{1}^{\prime}(z)}\right\} d \theta \\
& +\sigma \int_{\theta_{1}}^{\theta_{2}} \Re \frac{2 h^{\prime}(z)}{h(z)} d \theta+\beta_{1}\left(\theta_{2}-\theta_{1}\right) .
\end{aligned}
$$


For $h \in P(\beta)$, we observe that

$$
\begin{aligned}
\frac{\partial}{\partial \theta} \arg h\left(r e^{i \theta}\right) & =\frac{\partial}{\partial \theta} \Re\left\{-i \ln h\left(r e^{i \theta}\right)\right\} \\
& =\Re\left\{r e^{i \theta} \frac{h^{\prime}\left(r e^{i \theta}\right)}{h\left(r e^{i \theta}\right)}\right\} .
\end{aligned}
$$

Therefore

$$
\int_{\theta_{1}}^{\theta_{2}} \Re\left\{\frac{r e^{i \theta} h^{\prime}\left(r e^{i \theta}\right)}{h\left(r e^{i \theta}\right)}\right\} d \theta=\arg h\left(r e^{i \theta_{2}}\right)-\arg h\left(r e^{i \theta_{1}}\right),
$$

and

$$
\max _{h \in P(\beta)}\left|\int_{\theta_{1}}^{\theta_{2}} \mathfrak{R}\left\{\frac{r e^{i \theta} h^{\prime}\left(r e^{i \theta}\right)}{h\left(r e^{i \theta}\right)}\right\} d \theta\right|=\max _{h \in P(\beta)}\left|\arg h\left(r e^{i \theta_{2}}\right)-\arg h\left(r e^{i \theta_{1}}\right)\right| .
$$

We can write

$$
\frac{1}{1-\beta}[h(z)-\beta]=p(z), \quad p \in P,
$$

and for $|z|=r<1$, it is well known that

$$
\left|p(z)-\frac{1+r^{2}}{1-r^{2}}\right| \leq \frac{2 r}{1-r^{2}}
$$

From this, we have

$$
\left|h(z)-\frac{1+(1-2 \beta) r^{2}}{1-r^{2}}\right| \leq \frac{2(1-\beta) r}{1-r^{2}}
$$

Thus the values of $h$ are contained in the circle of Apollonius whose diameter is the line segment from $\frac{1-(1-2 \beta) r}{1+r}$ to $\frac{1+(1-2 \beta) r}{1-r}$ and has the radius $\frac{2(1-\beta) r}{1-r^{2}}$. So $|\arg h(z)|$ attains its maximum at points where a ray from origin is tangent to the circle, that is, when

$$
\arg h(z)= \pm \sin ^{-1}\left(\frac{2(1-\beta) r}{1-(1-2 \beta) r^{2}}\right) \text {. }
$$

From (3.3), we observe that

$$
\begin{aligned}
\max _{h \in P(\beta)}\left|\int_{\theta_{1}}^{\theta_{2}} \Re\left\{r e^{i \theta} \frac{h^{\prime}\left(r e^{i \theta}\right)}{h\left(r e^{i \theta}\right)}\right\} d \theta\right| & \leq 2 \sin ^{-1}\left(\frac{2(1-\beta) r}{1-(1-2 \beta) r^{2}}\right) \\
& =\pi-2 \cos ^{-1}\left(\frac{2(1-\beta) r}{1-(1-2 \beta) r^{2}}\right) .
\end{aligned}
$$

Also, for $\Psi_{1} \in C$,

$$
\int_{\theta_{1}}^{\theta_{2}} \mathfrak{R}\left\{1+r e^{i \theta} \frac{\Psi_{1}^{\prime \prime}\left(r e^{i \theta}\right)}{\Psi_{1}^{\prime}\left(r e^{i \theta}\right)}\right\} d \theta \geq 0
$$

Using (3.4) and (3.5) in (3.2), we obtain the required result. 
We note the following special cases:

1. For $k=0,0 \leq \theta_{1}<\theta_{2} \leq 2 \pi, z=r e^{i \theta}$, it follows from Theorem 3.2 that

$$
\int_{\theta_{1}}^{\theta_{2}} \Re\left\{1+\frac{z f^{\prime \prime}(z)}{f^{\prime}(z)}\right\} d \theta>-\pi \quad(z \in E) .
$$

This is a necessary and sufficient condition for $f$ to be close-to-convex (hence univalent) in $E$; see [7]. This also shows that $S T_{s}(\beta) \subset K$.

2. For $k=1 \int_{\theta_{1}}^{\theta_{2}} \Re\left\{1+\frac{z f^{\prime \prime}(z)}{f^{\prime}(z)}\right\} d \theta>-\frac{\pi}{2}$.

3. When $k \in[0,1]$, it is obvious that $\sigma \in(0,1]$. In this case, the class $k-S T_{s}(\beta)$ consists of strongly close-to-convex functions of order $\sigma$ in the sense of Pommerenke [20, 21].

Theorem 3.3 (Integral representation) Let $f \in k-S T_{s}(\beta)$. Then

$$
f^{\prime}(z)=\frac{1}{2} p(z) \exp \int_{0}^{z} \frac{1}{t}[p(t)+p(-t)-2] d t
$$

where $p \in P\left(p_{k, \beta}\right), z \in E$.

Proof Since $f \in k-S T_{s}(\beta)$, we can write

$$
\frac{2 z f^{\prime}(z)}{f(z)-f(-z)}=p(z), \quad p \in P\left(p_{k, \beta}\right)
$$

This gives us

$$
\frac{2[f(z)-f(-z)]^{\prime}}{f(z)-f(-z)}-\frac{1}{z}=\frac{1}{2}[p(z)-p(-z)-2]
$$

and the result follows when we integrate.

When $k=0, \beta=0$, we obtain the result for the class $S_{s}^{*}$ given in [5].

We now study the class $k-S T_{s}(\beta)$ under a certain integral operator.

Theorem 3.4 Let $g \in k-S T_{s}(\beta)$ and let for $m=1,2,3, \ldots, G$ be defined by

$$
G(z)=\frac{m+1}{2 z^{m}} \int_{0}^{z} t^{m-1}\{g(t)-g(-t)\} d t
$$

Then $G(z)$ belongs to $k-S T_{s}(\beta)$ in $E$.

Proof Let

$$
J(z)=\int_{0}^{z} t^{m-1} \frac{g(t)-g(-t)}{2} d t
$$

Since $g \in k-S T_{s}(\beta), \frac{1}{2}\{g(z)-g(-z)\} \in k-S T(\beta) \subset S^{*}\left(\beta_{1}\right) \subset S^{*}$, and $\beta_{1}=\frac{k+\beta}{k+1}$. Therefore it can easily be verified that $J(z)$ is $(m+1)$-valently starlike in $E$. 
We can write (3.6) as

$$
z^{m} G(z)=(m+1) J(z)
$$

and, differentiating logarithmically, we have

$$
\frac{z G^{\prime}(z)}{G(z)}=\frac{z J^{\prime}(z)-m J(z)}{J(z)}=\frac{N(z)}{D(z)}
$$

say, where $N(0)=D(0)=0$ and $D$ is $(m+1)$-valently starlike.

Let

$$
\frac{N(z)}{D(z)}=h(z)
$$

Then

$$
\begin{aligned}
\frac{N^{\prime}(z)}{D^{\prime}(z)} & =h(z)+\frac{z h^{\prime}(z)}{h_{0}(z)}, \quad h_{0}(z)=\frac{z D^{\prime}(z)}{D(z)} \in P \\
& =h(z)+H_{0}(z)\left(z h^{\prime}(z)\right), \quad H_{0}=\frac{1}{h_{0}} \in P .
\end{aligned}
$$

Since

$$
\begin{aligned}
\frac{N^{\prime}(z)}{D^{\prime}(z)} & =\frac{\left(z h^{\prime}(z)\right)^{\prime}-m J^{\prime}(z)}{J^{\prime}(z)} \\
& =\left\{\frac{\left(z J^{\prime}(z)\right)^{\prime}}{J^{\prime}(z)}-m\right\} \in P\left(p_{k, \beta}\right) .
\end{aligned}
$$

We now apply Lemma 2.2 to obtain

$$
\frac{N(z)}{D(z)}=\frac{z G^{\prime}(z)}{G(z)} \in P\left(p_{k, \beta}\right), \quad z \in E .
$$

This proves that $G \in k-S T(\beta)$ in $E$.

Theorem 3.5 Let $f, g \in k-S T_{s}(\beta)$ and let $F$ be defined by the following integral operator:

$$
F(z)=\left(\gamma+\frac{1}{\delta}\right) z^{1-\frac{1}{\delta}} \int_{0}^{z} t^{\frac{1}{\delta}-2}\left[\frac{f(t)-f(-t)}{2}\right]^{\frac{1}{1+\gamma}}\left[\frac{g(t)-g(-t)}{2}\right] d t
$$

where $z \in E, \delta>0, \gamma \geq 0$ and $\left[\frac{k(1+\gamma)}{k+1}+\left(\frac{1}{\delta}-1\right)\right]>\beta$. Then $F(z)$ belongs to $k-S T(\beta)$ for $z \in E$.

When $g(z)=z, \gamma=0$, we obtain a generalized form of the Bernardi operator; see [1]. Also for $g(z)=z, \gamma=0$, and $\delta=\frac{1}{2}$, we have the well-known integral operator studied by Libera [11] who showed that it preserves the geometric properties of convexity, starlikeness, and close-to-convexity.

Proof Let $\frac{f(z)-f(-z)}{2}=\Psi_{1}(z), \frac{g(z)-g(-z)}{2}=\Psi_{2}(z)$. Then $\Psi_{1}, \Psi_{2} \in k-S T(\beta)$ in $E$. We can write (3.8) as

$$
F(z)=\left(\gamma+\frac{1}{\delta}\right) z^{1-\frac{1}{\delta}} \int_{0}^{z} t^{\frac{1}{\delta}-2}\left(\Psi_{1}(t)\right)^{\frac{1}{1+\gamma}}\left(\Psi_{2}(t)\right) d t .
$$


Differentiating (3.9) logarithmically, and with $p(z)=\frac{z F^{\prime}(z)}{F(z)}$, we have

$$
\frac{\gamma}{1+\gamma} \frac{z \Psi_{1}^{\prime}}{\Psi_{1}(z)}+\frac{1}{1+\gamma} \frac{z \Psi_{2}^{\prime}}{\Psi_{2}(z)}=p(z)+\frac{z p^{\prime}(z)}{(1+\gamma) p(z)+\left(\frac{1}{\delta}-1\right)} .
$$

Since, for $i=1,2, \Psi_{i} \in k-S T(\beta), \frac{z \Psi_{1}^{\prime}(z)}{\Psi_{1}}=h_{1}(z), \frac{z \Psi_{2}^{\prime}(z)}{\Psi_{2}}=h_{2}(z)$ both belong to $P\left(p_{k, \beta}\right)$ in $E$, and $P\left(p_{k, \beta}\right)$ is a convex set. Therefore

$$
\left(\frac{\gamma}{1+\gamma} h_{1}(z)+\frac{1}{1+\gamma} h_{2}(z)\right) \in P\left(p_{k, \beta}\right), \quad z \in E
$$

From (3.10) and (3.11), it follows that

$$
\left(p(z)+\frac{z p^{\prime}(z)}{(1+\gamma) p(z)+\left(\frac{1}{\delta}-1\right)}\right) \prec p_{k, \beta}(z)
$$

We now apply Lemma 2.3 which gives us

$$
p(z) \prec q_{k, \beta}(z) \prec p_{k, \beta}(z)
$$

Thus $F \in k-S T(\beta)$ and the proof is complete.

\section{The class $k-U K_{s}(\beta)$}

Here we shall study some properties of the class $k-U K_{s}(\beta)$ which consists of $k$-uniformly close-to-convex functions.

Let $L(r, f)$ denote the length of the image of the circle $|z|=r$ under $f$. We prove the following.

Theorem 4.1 Let $f \in k-U K_{s}(\beta)$. Then, for $0<r<1, k \in[0,1]$,

$$
L(r, f)=O(1)\left(\frac{1}{1-r}\right)^{\sigma-\beta_{1}}, \quad \beta_{1}<\frac{\sigma}{2}
$$

where $\beta_{1}=\frac{k+\beta}{k+1}$ and $\sigma$ is given by (1.3), and $O(1)$ is a constant depending only on $k, \beta$.

Proof For $f \in k-U K_{s}(\beta)$, we can write

$$
z f^{\prime}(z)=\Psi(z) h^{\sigma}(z), \quad h \in P, \Psi \in S^{*}\left(\beta_{1}\right)
$$

and $\Psi(z)=\{g(z)-g(-z)\}, g \in k-S T_{s}(\beta)$.

Since $\Psi \in S^{*}\left(\beta_{1}\right)$ and is odd, there exists an odd starlike function $\Psi_{1}(z)$ such that

$$
\Psi(z)=z\left(\frac{\Psi_{1}(z)}{z}\right)^{1-\beta_{1}}=z\left(\frac{\Psi_{1}(z)}{z}\right)^{\frac{1-\beta_{1}}{k+1}}
$$

Thus, with $z=r e^{i \theta}$,

$$
L(r, f)=\int_{0}^{2 \pi}\left|z f^{\prime}(z)\right| d \theta=\int_{0}^{2 \pi}\left|z^{\beta_{1}}\left(\Psi_{1}(z)\right)^{1-\beta_{1}} h^{\sigma}(z)\right| d \theta
$$


and using Hölder's inequality, we have

$$
L(r, f) \leq 2 \pi r^{\beta_{1}}\left(\frac{1}{2 \pi} \int_{0}^{2 \pi}\left|\Psi_{1}(z)\right|^{(1-\beta)\left(\frac{z}{z-\sigma}\right)} d \theta\right)^{\frac{2-\sigma}{z}}\left(\frac{1}{2 \pi} \int_{0}^{2 \pi}|h(z)|^{2} d \theta\right)^{\frac{\sigma}{2}} .
$$

For $h \in P$, it is well known [20] that

$$
\frac{1}{2 \pi} \int_{0}^{2 \pi}|h(z)|^{2} d \theta \leq \frac{1+3 r^{2}}{1-r^{2}} .
$$

Using (4.3) and subordination for odd starlike functions in (4.2), it follows that

$$
\begin{aligned}
L(r, f) & \leq C\left(\beta_{1}, \sigma\right)\left(\frac{1}{1-r^{2}}\right)^{\left[\left(1-\beta_{1}\right)\left(\frac{2}{2-\sigma}\right)-1\right]\left[\frac{1+3 r^{2}}{1-r}\right]^{\frac{\sigma}{2}}} \\
& =O(1)\left(\frac{1}{1-r}\right)^{\sigma-\beta_{1}}
\end{aligned}
$$

where $C$ and $O(1)$ are constants depending only on $\beta_{1}$ and $\sigma$. This completes the proof.

We now discuss the growth rate of coefficients of $f \in k-U K_{s}(\beta)$.

Theorem 4.2 Let $f \in k-U K_{s}(\beta)$ and be given by (1.1). Then

$$
a_{n}=O(1) n^{\sigma-\beta_{1}-1}, \quad n \geq 1, \beta_{1}<\frac{\sigma}{2},
$$

where $O(1)$ is a constant depending only on $\sigma$ and $\beta_{1}$ and $\sigma, \beta_{1}$ are as given in Theorem 4.1.

Proof For $z=r e^{i \theta}, n \geq 1$, Cauchy's Theorem gives us

$$
\begin{aligned}
n\left|a_{n}\right| & =\frac{1}{2 \pi r^{n+1}}\left|\int_{0}^{2 \pi} z f^{\prime}(z) e^{-i n \theta} d \theta\right| \\
& \leq \frac{1}{2 \pi r^{n+1}} \int_{0}^{2 \pi}\left|z f^{\prime}(z)\right| d \theta \\
& =\frac{1}{2 \pi r^{n}} L(r, f) .
\end{aligned}
$$

With $r=\left(1-\frac{1}{n}\right)$, we use Theorem 4.1 and obtain the required result.

Theorem 4.3 Let $f \in k-U K_{s}(\beta)$ and let $F$ be defined by

$$
F(z)=\frac{m+1}{2 z^{m}} \int_{0}^{z} t^{m-1}\{f(t)-f(-t)\} d t .
$$

Then $F \in k-U K_{s}(\beta)$ in $E$. That is, the class $k-U K_{s}(\beta)$ is preserved under the integral operator (4.4).

Proof Since $f \in k-U K_{s}(\beta)$, we can write

$$
\left\{\frac{2 z f^{\prime}(z)}{g(z)-g(-z)}\right\} \in P\left(p_{k, \beta}\right), \quad g \in k-S T_{s}(\beta) \subset S_{S}^{*}\left(\beta_{1}\right) .
$$


Let $G(z)=\frac{1}{2}\left\{g_{1}(z)-g_{1}(-z)\right\}$ and be defined by (3.5). By Theorem 3.4, $g_{1} \in k-S T(\beta)$ and $G \in k-S_{s} T(\beta) \subset S_{s}^{*}\left(\beta_{1}\right)$. Let $G=z G_{1}^{\prime}$. Then we can write

$$
G_{1}^{\prime}(z)=\frac{1}{2}\left[z g_{1}(z)-g_{1}(-z)\right]^{\prime}, \quad G_{1} \in k-U C V_{s}(\beta) .
$$

Thus, from (4.4) and $g=z g_{1}^{\prime}, g_{1} \in C_{s}\left(\beta_{1}\right)$, we have

$$
\begin{aligned}
\frac{2 F^{\prime}(z)}{\left[g_{1}(z)-g_{1}(-z)\right]^{\prime}} & =\frac{z^{m}\{f(z)-f(-z)\}-m \int_{0}^{z} t^{m-1}\{f(t)-f(-t)\} d t}{z^{m}\left\{g_{1}(z)-g_{1}(-z)\right\}-m \int_{0}^{z} t^{m-1}\left\{g_{1}(t)-g_{1}(-t)\right\} d t} \\
& =\frac{N(z)}{D(z)},
\end{aligned}
$$

say. We note that $N(0)=D(0)=0$, and for $g_{1} \in C_{S}\left(\beta_{1}\right)$,

$$
\begin{aligned}
\frac{\left(z D^{\prime}(z)\right)^{\prime}}{D^{\prime}(z)} & =m+\frac{\left\{z\left[g_{1}(z)-g_{1}(-z)\right]^{\prime}\right\}^{\prime}}{\left\{g_{1}(z)-g_{1}(-z)\right\}^{\prime}} \\
& =m+h_{1}(z), \quad h_{1} \in P\left(\beta_{1}\right) .
\end{aligned}
$$

Since $P\left(\beta_{1}\right)$ is a convex set, $D \in C_{s}\left(\beta_{1}\right) \subset S^{*}$ in $E$. We thus have

$$
\frac{N^{\prime}(z)}{D^{\prime}(z)}=\frac{1}{2}\left[\frac{2 z f^{\prime}(z)}{\left[g_{1}(z)-g_{1}(-z)\right]^{\prime}}+\frac{2(-z) f^{\prime}(-z)}{\left[g_{1}(-z)-g_{1}(z)\right]^{\prime}}\right] \in P\left(p_{k, \beta}\right) .
$$

Now, using Lemma 2.2, it follows that

$$
\frac{N(z)}{D(z)}=\frac{2 F^{\prime}(z)}{\left(g_{1}(z)-g_{1}(-z)\right)^{\prime}} \in P\left(p_{k, \beta}\right) \quad \text { for } z \in E
$$

This proves that $F \in k-U K_{S}(\beta)$ in $E$.

We study a partial converse of the above result as follows.

Theorem 4.4 Let $\left(\frac{2 z f^{\prime}(z)}{g(z)-g(-z)}\right) \prec p_{k}(z)$ in E and let

$$
F_{1}(z)=\frac{1}{1+m} z^{1-m}\left(z^{m} f(z)\right)^{\prime}, \quad m=1,2,3, \ldots
$$

Then $F_{1} \in K_{s}$ for $|z|<r_{1}$, where

$$
r_{1}=\left\{\frac{m+1}{\left(2-\beta_{1}\right)+\sqrt{\left(z-\beta_{1}\right)^{2}+(m+1)\left(m-1+2 \beta_{1}\right)}}\right\}, \quad \beta_{1}=\frac{k+\beta}{k+1} .
$$

Proof We shall need the following well-known results for $p \in P(\alpha), 0 \leq \alpha<1$; see [4]:

$$
\begin{aligned}
& \frac{1-(1-2 \alpha) r}{1+r} \leq|p(z)| \leq \frac{1+(1-2 \alpha) r}{1-r}, \\
& \left|p^{\prime}(z)\right| \leq \frac{2[\Re p(z)-\alpha]}{1-r^{2}} .
\end{aligned}
$$


Since $f \in k-U K_{s}(\beta)$, there exists $g \in S_{s}^{*}\left(\beta_{1}\right)$ such that, for $z \in E$.

$$
\left(\frac{2 z f^{\prime}(z)}{g(z)-g(-z)}\right)=p(z), \quad p \in P\left(p_{k}\right) \subset P(\alpha), \alpha=\frac{k}{k+1} .
$$

From (4.5), we have

$$
F_{1}(z)=\frac{1}{1+m}\left[m f(z)+z f^{\prime}(z)\right]
$$

and this gives us

$$
\begin{aligned}
\frac{2 z F_{1}^{\prime}(z)}{g(z)-g(-z)} & =\frac{1}{m+1}\left[\frac{2 m f^{\prime}(z)}{g(z)-g(-z)}+\frac{2 z\left(z f^{\prime}(z)\right)^{\prime}}{g(z)-g(-z)}\right] \\
& =\frac{1}{m+1}\left[m p(z)+z p^{\prime}(z)+p(z) h(z)\right],
\end{aligned}
$$

where

$$
h(z)=\frac{z \Psi^{\prime}(z)}{\Psi(z)} \in P\left(\beta_{1}\right), \quad \Psi(z)=g(z)-g(-z)
$$

Now, using (4.7) and (4.8), we have

$$
\begin{aligned}
\Re\left\{\frac{2 z F_{1}^{\prime}(z)}{g(z)-g(-z)}\right\} & \geq \frac{(\Re p(z)-\alpha)}{1+m}\left\{m+\frac{1-\left(1-2 \beta_{1}\right) r}{1+r}-\frac{2 r}{1-r^{2}}\right\} \\
& =\frac{\Re p(z)-\alpha}{1+m}\left[\frac{T(r)}{1-r^{2}}\right],
\end{aligned}
$$

where

$$
T(r)=(m+1)-2\left(2-\beta_{1}\right) r+\left(-m-2 \beta_{1}+1\right) r^{2} .
$$

We note that $T(0)=1+m>0$ and $T(1)=-3<0$. So there exists $r_{1} \in(0,1)$. The right hand side of (4.9) is positive for $|z|<r_{1}$, where $r_{1}$ is given by (4.6). This implies that $F \in K_{s}$ for $|z|<r_{1}$ and the proof is complete.

We have the following special cases.

1. For $k=0=\beta, f \in K_{s}$. Then $F_{1}$, defined by (4.5) belongs to $K_{s}$ for $|z|<r_{0}=\frac{1+m}{2+\sqrt{3+m^{2}}}$.

2. When $m=1$ and $\beta_{1}=0$ (that is, $k=0=\beta$ ), then $F_{1}(z)=\frac{(z f(z))^{\prime}}{2}$ belongs to the same class for $|z|<\frac{1}{2}$. This result has been proved by Livingston [12] for convex and starlike functions. 


\section{Acknowledgements}

The author would like to thank editor and anonymous referee for their valuable suggestions. The author is grateful to Dr SM Junaid Zaidi, Rector, COMSATS Institute of Information Technology, Pakistan for providing an excellent research and academic environment. This research is supported by HEC NRPU project No: 20-1966/R\&D/11-2553, titled, Research unit od Academic Excellence in Geometric Function Theory and Applications.

Received: 28 March 2014 Accepted: 13 June 2014 Published: 22 Jul 2014

\section{References}

1. Bernardi, SD: Convex and starlike univalent functions. Trans. Am. Math. Soc. 135, 429-446 (1969)

2. Das, RN, Singh, P: On subclasses of Schlicht mappings. Indian J. Pure Appl. Math. 8, 864-872 (1977)

3. Goodman, AW: On uniformly starlike functions. J. Math. Anal. Appl. 155, 364-370 (1991)

4. Goodman, AW: Univalent Functions, Vol I \& II. Polygonal Publishing House, Washington (1983)

5. Kanas, S: Differential subordination related to conic sections. J. Math. Anal. Appl. 317(2), 650-658 (2006)

6. Kanas, S: Techniques of differential subordination for domains bounded by conic sections. Int. J. Math. Math. Sci. 38 2389-2400 (2003)

7. Kanas, S, Lecko, A, Moleda, A: Certain generalization of the Sakaguchi lemma. Folia Sci. Univ. Tech. Resov. 38, 35-42 (1987)

8. Kanas, S, Sugawa, T: On conformal representation of the interior of an ellipse. Ann. Acad. Sci. Fenn., Math. 31, 329-348 (2006)

9. Kanas, S, Wisniowska, A: Conic regions and k-uniform convexity. J. Comput. Appl. Math. 105, 327-336 (1999)

10. Kaplan, W: Close-to-convex Schlicht functions. Mich. Math. J. 1, 169-185 (1952)

11. Libera, RJ: Some classes of regular univalent functions. Proc. Am. Math. Soc. 16, 755-758 (1965)

12. Livingston, AE: On the radius of univalence of certain analytic functions. Proc. Am. Math. Soc. 17, $352-359$ (1966)

13. Ma, W, Minda, D: Uniformly convex functions. Ann. Pol. Math. 57(2), 165-175 (1992)

14. Ma, W, Minda, D: Uniformly convex functions II. Ann. Pol. Math. 58(3), 275-285 (1992)

15. Miller, SS, Mocanu, PT: Univalent solution of Briot-Bouquet differential equations. J. Differ. Equ. 56, 297-308 (1985)

16. Noor, Kl, Noor, MA: Higher order close-to-convex functions related with conic domain. Appl. Math. Inf. Sci. 8(5), 2455-2463 (2014)

17. Noor, Kl, Ahmad, QZ, Noor, MA: On some subclasses of analytic functions defined by fractional derivative in the conic regions. Appl. Math. Inf. Sci. (2014/15)

18. Noor, Kl, Fayyaz, R, Noor, MA: Some classes of k-uniformly functions with bounded radius rotation. Appl. Math. Inf. Sci. $8(2), 1-7(2014)$

19. Noor, Kl, Noor, MA, Murtaza, R: Inclusion properties with applications for certain subclasses of analytic functions. Appl. Math. Inf. Sci. (2014/15)

20. Pommerenke, C: On close-to-convex analytic functions. Trans. Am. Math. Soc. 14, 176-186 (1965)

21. Pommerenke, C: On starlike and close-to-convex functions. Proc. Lond. Math. Soc. 3, 290-304 (1963)

22. Ronning, F: On starlike functions associated with parabolic regions. Ann. Univ. Mariae Curie-Skłodowska, Sect. A 45, 117-122 (1991)

23. Ronning, F: Uniformly convex functions and a corresponding class of starlike functions. Proc. Am. Math. Soc. 118 189-196 (1993)

24. Sakaguchi, K: On a certain univalent mapping. J. Math. Soc. Jpn. 11, $72-73$ (1959)

25. Stankiewicz, J: Some remarks on functions starlike with respect to symmetrical points. Ann. Univ. Mariae Curie-Skłodowska. Sect. A 19, 53-59 (1965)

10.1186/1029-242X-2014-254

Cite this article as: Noor: On uniformly univalent functions with respect to symmetrical points. Journal of Inequalities and Applications 2014, 2014:254

\section{Submit your manuscript to a SpringerOpen ${ }^{\circ}$ journal and benefit from:}

- Convenient online submission

- Rigorous peer review

- Immediate publication on acceptance

- Open access: articles freely available online

- High visibility within the field

- Retaining the copyright to your article 